\title{
Pigeons transfer between conditional discriminations with differential outcomes in the absence of differential-sample-responding cues
}

\author{
LOU M. SHERBURNE and THOMAS R. ZENTALL \\ University of Kentucky, Lexington, Kentucky
}

\begin{abstract}
Pigeons were trained to perform two independent zero-delay conditional discriminations involving the same differential outcomes (i.e., food vs. a feeder light) and were then tested by replacing the samples from one of the tasks with those from the other. Differential responding to the two samples was required in Phase 1, in Phase 2, in neither phase, or in both phases. Half the pigeons in each group were then tested with associations that were either consistent with the presumed outcome expectancies established during Phases 1 and 2 (positive transfer condition) or inconsistent with those expectancies (negative transfer condition). The magnitude of the transfer effect was largest in the group that could use differential sample responding as a cue to mediate transfer, but significant transfer effects were also found in the groups that could use only outcome expectancies. Thus, differential sample responding contributes to, but does not account for, the differential-outcomes effect.
\end{abstract}

In a standard two-choice conditional discrimination, presentation of one sample signals reinforcement for a response to one of the comparisons, whereas presentation of the other sample signals reinforcement for a response to the alternative comparison. Animals learn such discriminations more rapidly and perform them to a higher level (especially when delays are inserted between the offset of the sample and the onset of the comparisons) when the comparison responses associated with the different samples are reinforced with outcomes that are either qualitatively or quantitatively different (Brodigan \& Peterson, 1976; Trapold, 1970).

The results of transfer-of-control experiments suggest that this differential-outcomes effect (DOE) arises because the animals use sample-generated expectancies of trial outcomes as additional cues for correct responding. Peterson (1984), for example, trained pigeons to perform a conditional discrimination involving differential outcomes for correct responding (i.e., food vs. no food) and, off baseline, trained them on a simple successive discrimination between two other stimuli associated with the same differential outcomes. When the samples were replaced with the stimuli from the simple successive discrimination, the pigeons showed excellent transfer of perfor-

This research was supported by National Science Foundation Grants BNS-8418275 and BNS-9019080 and National Institute of Mental Health Grant 45979 to T. R. Zentall and P. J. Urcuioli. We thank Peter J. Urcuioli for suggesting the Diff-Resp/Nondiff-Resp and Nondiff-Resp/Diff-Resp groups and Karen L. Roper for her general assistance with this research. Correspondence concerning this article should be addressed to L. M. Sherburne, who is now at the Department of Psychology, Wabash College, Crawfordsville, IN 47933 (e-mail: sherburl@scholar.wabash.edu).

-Accepted by previous editor, Vincent M. LoLordo mance. Although the pigeons had no experience with the test stimuli as cues for comparison choice, they were able to perform correctly, apparently, by using the anticipation of food as a cue for choosing the food-associated comparison and anticipation of the absence of food as a cue for choosing the other comparison.

An alternative account of the DOE is suggested by research which shows that the topography of pigeons' responding to a stimulus differs as a function of the type of reinforcement with which it is associated (Jenkins \& Moore, 1973), and that pigeons can use differential sample responding to mediate correct comparison choice in conditional discriminations (Cohen, Looney, Brady, \& Aucella, 1976; Urcuioli, 1985; Urcuioli \& Honig, 1980; Zentall, Hogan, Howard, \& Moore, 1978). Research has also shown that pigeons tend to respond differentially to samples that predict different outcomes (e.g., food vs. water, Brodigan \& Peterson, 1976; food vs. no food, Urcuioli, 1990). Samples associated with food outcomes, for example, engender a high rate of pecking, whereas samples associated with no-food outcomes are pecked very little. Thus, it is possible that differential sample responding resulting from the anticipated outcomes, rather than the anticipated outcomes themselves, contributes to or is even responsible for the DOE.

Urcuioli and DeMarse (1994) have, in fact, reported evidence that cues produced by differential sample responding alone are sufficient to mediate transfer when samples from a differential-outcomes conditional discrimination are replaced with other stimuli associated (off baseline) with differential sample responding, but with nondifferential outcomes. In one of their experiments, for example, pigeons were first trained on a conditional discrimination involving food versus no-food outcomes. By the end of training, the pigeons readily pecked the food-associated 
sample, but not the no-food-associated sample. The pigeons were then trained on a simple successive discrimination with new stimuli, one of which they were required to peck for reinforcement (fixed interval, FI, $3 \mathrm{sec}$ ) and the other, to refrain from pecking to receive the same reinforcement (differential reinforcement of other behavior, DRO, $3 \mathrm{sec})$. Thus, the same food outcomes were associated with both of these stimuli. When the stimuli from the simple discrimination replaced the samples from the differential-outcomes task, significant transfer was found. These results suggest that differential sample responding may be sufficient to account for the effects found in transfer-of-control studies in which both sets of samples are associated with differential outcomes.

In the absence of evidence that such transfer effects can be obtained without overtly differential sample responding, it would seem more parsimonious to attribute the DOE to observable differences in behavior, than to unobservable differences in outcome anticipation. On the other hand, although Urcuioli and DeMarse's (1994) results suggest that the differential sample responding generated by differential outcomes is sufficient to guide pigeons' comparison choice, it is not clear whether differential sample responding is a necessary condition for transfer between two differential-outcomes tasks.

Evidence that the DOE cannot be reduced to overt behavioral mediation is suggested by research in which the differential sample responding normally produced by differential outcomes has been eliminated. When Delong and Wasserman (1981) had pigeons respond similarly to both samples in a successive conditional discrimination, those trained with differential outcomes still showed better long-delay performance than did those trained with nondifferential outcomes. Furthermore, Zentall and Sherburne (1994) have recently reported findings which suggest that the memory codes generated by differential outcomes (accompanied by differential sample responding) differ qualitatively from those generated by differential sample responding alone. Pigeons trained on a conditional discrimination with differential sample responding (to peck one sample and not to peck the other) but nondifferential outcomes showed flat delay functions on no-peck sample trials and steep delay functions on peck sample trials. Flat, parallel delay functions were found, however, when differential outcomes (i.e., food vs. no food) accompanied the differential sample responding. In addition, pigeons trained to peck both samples similarly, but reinforced with differential outcomes, showed flat, symmetrical delay functions indistinguishable from those seen in pigeons trained with both differential sample responding and differential outcomes. Although a theoretical account of the symmetries and asymmetries of delay functions following conditional discrimination training is beyond the scope of the present article (see Sherburne \& Zentall, 1993), these results suggest that in a differentialoutcomes task, differential sample responding alone does not control comparison choice.

The present study was designed to determine whether differential outcomes, in the absence of differential sam- ple responding, could provide cues sufficient to mediate transfer when samples were interchanged between two independent conditional discriminations. A second goal of this experiment was to determine whether differential sample responding contributes to the magnitude of transfer.

To separate the anticipation of differential outcomes from the differential sample responding that these outcomes normally produce, a procedure used by Zentall and Sherburne (1994) was employed in which nondifferential sample responding accompanied differential outcomes. To get pigeons to respond similarly to samples associated with food outcomes and samples associated with no-food outcomes, single-stimulus trials were included in which pecks to the "no-food" sample were followed immediately by food, rather than by comparisons (cf. Delong \& Wasserman, 1981). Because these single-stimulus trials were randomly interspersed among the matching trials, on any given no-food trial, the trial outcome could be accurately predicted only after sample termination. Thus, although both samples were associated with food outcomes, the absence of reinforcement following the no-food sample (as well as presentation of the comparison stimuli) signaled a conditional discrimination trial with a no-food outcome.

A second important aspect of the present procedure is the assessment of transfer effects by comparison of performance in positive versus negative transfer conditions. That is, in the transfer phase of the experiment, some animals were reinforced for comparison responses consistent with the presumed outcome expectancies (positive transfer), whereas others were reinforced for comparison responses inconsistent with the presumed outcome expectancies (negative transfer; see Edwards, Jagielo, Zentall, \& Hogan, 1982). To properly assess the contribution of outcome expectancies established during training, the transfer phase was conducted with nondifferential outcomes for correct comparison choices. Thus, with this procedure, transfer effects based on differential outcome anticipations should be transient, and matching accuracy in the positive transfer conditions might actually be expected to decline with additional transfer task experience, as the differential outcome anticipations are disconfirmed.

A summary of the design of the experiment is presented in Table 1. In Phase 1, for half of the pigeons, responding to a dot comparison (D) on red-sample (R) trials was followed by a food outcome $(F)$, whereas responding to a circle comparison $(\mathrm{C})$ on green-sample $(\mathrm{G})$ trials was followed by a no-food outcome (NF). For the remaining pigeons (not shown in Table 1), the roles of the C and D comparisons were interchanged. In Phase 2, for all pigeons, responding to a vertical-line comparison (V) on blue-sample (B) trials was followed by $\mathrm{F}$ and responding to a horizontal-line comparison $(\mathrm{H})$ on white-sample (W) trials was followed by NF. Thus, all pigeons received differential-outcomes training in both training phases. In the transfer phase, for all pigeons, responding to $\mathrm{C}$ on $\mathrm{B}$ sample trials and to $\mathrm{D}$ on W-sample trials was followed by nondifferential outcomes. For half of the pigeons (positive 
Table 1

Experimental Design

\begin{tabular}{llll}
\hline \multicolumn{1}{c}{ Group } & \multicolumn{1}{c}{ Phase 1} & \multicolumn{1}{c}{ Phase 2 } & Positive Transfer \\
\hline Diff-Resp/Diff-Resp & R-FR10 $\rightarrow$ C & B-FR10 $\rightarrow$ V & B-FR10 $\rightarrow$ C \\
& G-DRO6s $\rightarrow$ D & W-DRO6s $\rightarrow$ H & W-DRO6s $\rightarrow$ D \\
Diff-Resp/Nondiff-Resp & R-FR10 $\rightarrow$ C & B-FR 10 $\rightarrow$ V & B-FR10 $\rightarrow$ C \\
& G-DRO6s $\rightarrow$ D & W-FR10 $\rightarrow$ H & W-FR10 $\rightarrow$ D \\
Nondiff-Resp/Diff-Resp & R-FR10 $\rightarrow$ C & B-FR10 $\rightarrow$ V & B-FR10 $\rightarrow$ C \\
& G-FR10 $\rightarrow$ D & W-DRO6s $\rightarrow$ H & W-DRO6s $\rightarrow$ D \\
Nondiff-Resp/Nondiff-Resp & R-FR10 $\rightarrow$ C & B-FR10 $\rightarrow$ V & B-FR10 $\rightarrow$ C \\
& G-FR10 $\rightarrow$ D & W-FR10 $\rightarrow$ H & W-FR10 $\rightarrow$ D \\
\hline
\end{tabular}

Note- $R$, red; G, green; B, blue; W, white; $C$, circle; $D$, dot; V, vertical; $H$, horizontal. FR, fixed ratio; DRO, differential reinforcement of other behavior. In Phases 1 and 2, all pigeons were trained with differential outcomes (food, $F$, for correct comparison responses after $\mathrm{R}$ and $\mathrm{B}$; no food, NF, for correct comparison responses after $\mathrm{G}$ and $\mathrm{W}$ ). In the transfer phase, however, all pigeons had nondifferential outcomes (half of all correct comparison responses were reinforced with $F$ ). For half the pigeons in each group, Phase 1 training involved $\mathrm{R} \rightarrow \mathrm{D}$ and $\mathrm{G} \rightarrow \mathrm{C}$ associations (not shown). These pigeons constituted the negative transfer conditions in each group.

transfer), the sample-comparison mapping in transfer should have produced outcome anticipations consistent with those presumably acquired during training. For the remaining pigeons (negative transfer), the mapping of samples and comparisons onto outcomes should have produced outcome anticipations inconsistent with those presumably acquired during training.

The four groups differed according to whether they were trained to respond differentially (Diff-Resp) to the two samples, or not (Nondiff-Resp), in each phase. If pigeons use differential sample responding as the basis for comparison choice, the only group that should show a transfer effect is the one that responded to the samples differentially in both training phases (and the transfer phase). For the other three groups, either differential responding to the samples did not occur in the transfer phase or the comparisons used in the transfer phase had not appeared in training with samples to which there was differential sample responding.

\section{METHOD}

\section{Subjects}

The subjects were 32 White Carneaux pigeons (retired breeders, 5-8 years old) purchased from the Palmetto Pigeon Plant (Sumter, SC). Sixteen of the animals had had experience on a simple simultaneous discrimination task involving hues, 8 had had experience on a nondifferential-outcomes conditional discrimination, and 8 were experimentally naive. Prior to the start of the experiment, the pigeons were assigned to conditions such that previous training experience was balanced across groups. Purina Pro Grains, mostly provided during experimental sessions, maintained the pigeons at $80 \%-85 \%$ of their free-feeding weights. The pigeons were housed in individual wire mesh cages with water and grit freely available. The colony was maintained under a 12:12-h light:dark cycle.

\section{Apparatus}

Experimental sessions were conducted in two BRS/LVE (Beltsville, $M D$ ) operant chambers. One of the chambers measured $32 \mathrm{~cm}$ high, $28 \mathrm{~cm}$ from the front panel to the back wall, and $35 \mathrm{~cm}$ across the front panel. Stimuli were projected on three horizontally aligned circular response keys $(2.5 \mathrm{~cm}$ in diameter and $8.5 \mathrm{~cm}$ between centers of adjacent keys) by means of in-line stimulus projectors (In- dustrial Electronics Engincering, Series 10, Van Nuys, CA) mounted behind each key. The center key could be illuminated red, green, blue, or white (Kodak Wratten filters Nos. 26, 60, and 38a, or no filter, respectively). A white circle (C, $16 \mathrm{~mm}$ outside diameter, $13 \mathrm{~mm}$ inside diameter), a white dot (D, $5 \mathrm{~mm}$ diameter), three white vertical lines ( $V$, each $13 \mathrm{~mm}$ long $\times 3 \mathrm{~mm}$ wide and separated by $3 \mathrm{~mm}$ ), or three white horizontal lines ( $\mathrm{H}$, same dimensions as $\mathrm{V}$ ), presented on a black background, could be projected onto each of the side keys. The feeder was mounted behind the front panel such that, when raised, it was lit and accessible through a $5.1 \times 5.7 \mathrm{~cm}$ aperture centered horizontally on the panel. The bottom edge of the aperture was $7 \mathrm{~cm}$ above the wire mesh floor. A shielded houselight (GE 1820 lamp) was mounted $4 \mathrm{~cm}$ above the response keys.

The second chamber was similar to the first except that the three response keys were spaced $6 \mathrm{~cm}$ apart (center to center). In each chamber, extraneous sounds were masked by white noise at $72 \mathrm{~dB}$ presented through a speaker located on the left front wall of the chamber and by the sound of an exhaust fan mounted on the exterior of the chamber. Experimental contingencies and data recording were controlled by microcomputers (Apple IIe) located in an adjacent room.

\section{Procedure}

Preliminary training. Prior to pretraining, each of the pigeons was assigned to one of the two test chambers and to one of the eight groups resulting from the factorial combination of differential versus nondifferential sample responding (Diff-Resp vs. Nondiff-Resp) in Phase 1, Diff-Resp versus Nondiff-Resp in Phase 2, and positive versus negative transfer conditions. Whether a pigeon was required, during pretraining, to peck $R$ or both $R$ and $G$, was determined by its Phase 1 group assignment.

The birds that were experimentally naive were first trained to eat from the grain feeder and were then shaped by the method of successive approximations to peck either one or both of the two centerkey stimuli that would serve as samples in the first phase of the experiment ( $R$ and $G$ ). All 32 subjects were then exposed to 24-trial training sessions in which $R$ or $G$, on alternating trials (Phase 1 Nondiff-Resp birds), or R only (Phase 1 Diff-Resp birds) was presented on the center key and the fixed ratio (FR) required to the stimuli was gradually increased from 1 to 10 . End-of-trial reinforcement consisted of a 3-sec presentation of mixed grain. The houselight, which was otherwise off, was lit during the $10-\mathrm{sec}$ intertrial intervals (ITIs). Sample pretraining was continued for each pigeon until it had completed 24 FR 10 trials with each of the sample stimult it would be required to peck in Phase 1. All pigeons were then exposed to a 24-trial session in which they were required to respond once (FRI) 
to each presentation of the side-key stimuli that would serve as Phase I comparisons. Each of the comparison stimuli ( $C$ on the left, $\mathrm{C}$ on the right, $\mathrm{D}$ on the left, and D on the right) was presented six times, in random order.

Phase 1 training. For all pigeons, a trial began with presentation of either $\mathrm{R}$ or $\mathrm{G}$ on the center key. On R-sample trials, 10 responses were required to terminate the sample (FR10) and produce the $C$ and $\mathrm{D}$ comparisons on the side keys. For the 16 Diff-Resp birds, the response requirement on G-sample trials was DRO $6 \mathrm{sec}$ (i.e., these birds had to withhold sample responses for $6 \mathrm{sec}$ ). For the 16 Nondiff-Resp birds, the response requirement on G-sample trials was the same as that on R-sample trials, FR10. For half the pigeons in each group, on R-sample trials, responses to $C$ were reinforced with a 1.5 $\mathrm{sec}$ presentation of mixed grain (F) illuminated by the feeder light, and, on G-sample trials, responses to D were reinforced with a 1.5 sec presentation of the feeder light alone (NF). For the remaining pigeons, the sample-comparison associations were reversed (i.e., responses to $\mathrm{D}$ were followed by $\mathrm{F}$ on $\mathrm{R}$-sample trials, and responses to $\mathrm{C}$ were followed by NF on G-sample trials). Reinforcement of correct responses occurred during the first $1.5 \mathrm{sec}$ of the 10 -sec houselight-illuminated ITI. Incorrect comparison responses resulted in the ITI alone and repetitions of the trial, until a correct response was made (up to five repetitions of each trial). This correction procedure was used to encourage correct responding to the NF-associated comparison.

For all pigeons, the daily sessions were conducted 6 days a week and included 96 conditional discrimination trials (excluding repetition trials) balanced for sample hue and position of the correct comparison. In addition, for Nondiff-Resp birds (those required to peck the NF-associated sample), each session included 24 additional single-stimulus $\mathrm{G}$ trials in which the 10th sample peck did not produce comparisons but instead resulted in an immediate 1.5 -sec presentation of mixed grain. For the Diff-Resp birds, the 96-trial sessions were balanced over blocks of 8 trials; for the Nondiff-Resp birds, the 120-trial sessions were balanced over blocks of 10 trials. Two sequences of trial order were presented in double-alternating order over sessions.

Phase 2 training. After a pigeon had completed two consecutive Phase 1 sessions during which performance was at least $90 \%$ correct (excluding repetition trials), it began Phase 2 training. Phase 2 training was similar to that of Phase 1 except that the sample stimuli were $B$ and $W$ and the comparison stimuli were $V$ and $H$. Half the pigeons from each of the four Phase 1 groups (Diff-Resp, with $R$ associated with either $C$ or $D$, and Nondiff-Resp, with $R$ associated with either C or D) were required to respond differentially to the samples in Phase 2 (Diff-Resp, FR10 on B trials and DRO 6 sec on W trials). The remaining pigeons were required to respond nondifferentially to the samples (Nondiff-Resp, FR 10 on both B and W trials). For all pigeons, a response to $\mathrm{V}$ was reinforced with $1.5 \mathrm{sec}$ of mixed grain on B-sample trials, and a response to $H$ was reinforced with a 1.5sec presentation of the feeder light on W-sample trials. Each daily session was followed by a 24-trial Phase 1 reminder session. In all other respects, Phase 2 training was as described for Phase 1.

The groups resulting from manipulation of sample-response requirements in the training phases are designated by Phase 1 and Phase 2 response requirements, respectively. For example, the Nondiff-Resp/Diff-Resp group refers to those birds required to respond nondifferentially to the samples in Phase 1 and differentially to the samples in Phase 2.

Transfer. Testing began for each pigeon when it had completed two consecutive Phase 2 sessions during which matching accuracy was at least $90 \%$ correct and accuracy on the Phase 1 reminder trials was also at least $90 \%$ correct on the last session. In any case, pigeons were not tested until performance on both Phase 2 and reminder trials was at least $90 \%$ correct for one day.

All pigeons were tested with Phase 2 samples (B and $\mathrm{W}$, to which responses were required as trained in Phase 2) followed by a choice between Phase 1 comparisons ( $C$ and $D$ ). In the positive transfer condition, the associations reinforced in the transfer phase were those consistent with the presumed outcome anticipations established during training. In the negative transfer condition, the transfer phase associations were inconsistent with those presumed outcome anticipations. In the transfer phase, for all groups, correct comparison choices were reinforced with nondifferential outcomes. For all pigeons, responses to $\mathrm{C}$ were correct following $\mathrm{B}$ samples and responses to $\mathrm{D}$ were correct following $\mathrm{W}$ samples. On a random half of the trials, correct responses were followed by F. On the remaining trials, they were followed by NF. Incorrect comparison responses were followed by the ITI alone. The correction procedure was not used during the test phase. Test sessions consisted of 96 test trials for Diff-Resp/Diff-Resp and Nondiff-Resp/Diff-Resp pigeons, and 120 trials for Diff-Resp/Nondiff-Resp and Nondiff-Resp/Nondiff-Resp pigeons (including 24 single-stimulus trials as in training). All pigeons were given a minimum of five transfer sessions.

\section{RESULTS}

Analyses of variance (ANOVAs) were performed on the data, using the .05 significance level.

\section{Acquisition of the Training Phases}

One-way ANOVAs were performed on the sessions-tocriterion data from each of the training phases to determine whether pigeons' differential sample responding facilitated acquisition of the differential-outcomes task. Whether the sample-response requirement was differential or not did not appear to affect the rate of acquisition in either Phase 1 or Phase 2 (both $F \mathrm{~s}<1$ ).

\section{Transfer Phase: First Session}

The first-session transfer data appear in Figure 1. Within each of the four groups formed by the combinations of Phase 1 and Phase 2 response requirements, pi-

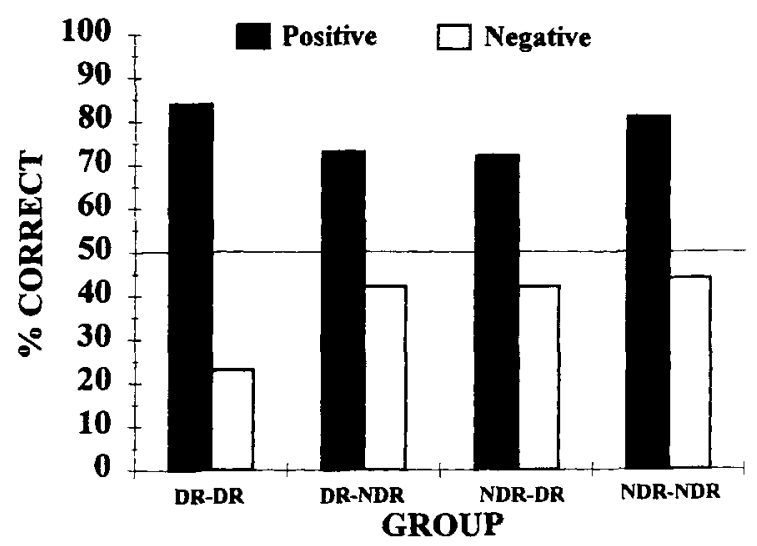

Figure 1. Matching accuracy on the first transfer session. DR indicates that differential sample responding $(10$ responses to one sample, the absence of responding to the other) was required either in Phase 1 (left of dash) or in Phase 2 (right of dash). NDR indicates that nondifferential sample responding $(10$ responses to both samples) was required. In the positive transfer condition, the hypothesized outcome expectancies established during training were consistent with accurate responding. In the negative transfer condition, they were not (see Table 1). 
geons in the positive transfer condition performed at a higher level than did those in the negative transfer condition. This effect was largest in the Diff-Resp/Diff-Resp group. These results were confirmed by a three-way ANOVA performed on the first-session data, with transfer condition (positive vs. negative) sample-response requirement in Phase 1 (differential vs. nondifferential) and sample-response requirement in Phase 2 (differential vs. nondifferential) as variables. The analysis revealed that performance in the positive transfer condition, overall (mean $=77.4 \%$ correct), was significantly higher than performance in the negative transfer condition [mean $=$ $37.8 \%$ correct; $F(1,24)=136.84$ ]. The overall level of performance (i.e., pooled over transfer condition) did not differ significantly among the four groups, however, as a function of sample-response requirement in Phase 1 $[F(1,24)=1.57]$, sample response requirement in Phase 2 $[F(1,24)=2.18]$, or their interaction $(F<1)$.

Although neither the Phase 1 sample-response requirement nor the Phase 2 sample-response requirement alone interacted significantly with the transfer effect $[F(1,24)=$ 3.40 and 2.40 , respectively], the three-way interaction was significant $[F(1,24)=7.66]$. Thus, the magnitude of the transfer effect depended on the combination of Phase 1 and Phase 2 sample-response requirements. A planned comparison revealed that the transfer effect was significantly larger in the group that could use differential sample responding to guide comparison choice in test (DiffResp/Diff-Resp group; means $=83.5 \%$ vs. $23.0 \%$ correct for positive and negative transfer conditions, respectively) than in the groups that could not do so [Diff-Resp/Nondiff-Resp, Nondiff-Resp/Diff-Resp, and Nondiff-Resp/ Nondiff-Resp; $F(1,28)=13.41$ ]. Nevertheless, the transfer effects found in the groups in which differential sample responding could not mediate test performance (DiffResp/Nondiff-Resp, Nondiff-Resp/Diff-Resp, and NondiffResp/Nondiff-Resp) were substantial as well [combined means $=75.3 \%$ vs. $42.7 \%$ correct, for positive vs. negative transfer conditions, respectively; $F(1,20)=61.10$ ].

The magnitude of the transfer effect in the group that responded nondifferentially to the samples in both Phases 1 and 2 (Nondiff-Resp/Nondiff-Resp, $81.2 \%$ vs. $43.8 \%$ correct for positive and negative transfer conditions, respectively) did not differ significantly from the transfer effect in the groups that responded nondifferentially to the samples in one phase and differentially in the other [DiffResp/Nondiff-Resp and Nondiff-Resp/Diff-Resp; $F(1,20)$ $=1.17]$. Nor did the magnitude of the transfer effect differ significantly between groups Diff-Resp/Nondiff-Resp and Nondiff-Resp/Diff-Resp ( $73.2 \%$ vs. $42.0 \%$ correct and $71.5 \%$ vs. $42.2 \%$ correct for Diff-Resp/Nondiff-Resp and Nondiff-Resp/Diff-Resp positive vs. negative transfer conditions, respectively; $F<1$ ).

Thus, given that the pigeons were required to respond nondifferentially to samples in at least one of the training phases, it did not appear to make a difference whether the nondifferential responding was required in the Phase 1 task, in the Phase 2 task, or in both. Differential sample responding can effectively mediate comparison choice only if the transfer task involves both samples and comparisons that have appeared earlier in the context of differential sample responding.

Evidence that pigeons tested with nondifferential sample responding were, in fact, responding nondifferentially to the samples was obtained by analyzing the durations of the two sample types (i.e., the time needed to complete the FR10) during the first transfer session. For the NondiffResp groups the mean duration of the sample previously associated with the food outcome (mean $=4.84 \mathrm{sec}$ ) was virtually the same as that of the sample previously associated with the no-food outcome on conditional discrimination trials $($ mean $=4.82 \mathrm{sec} ; F<1)$.

\section{Transfer Phase Acquisition}

Matching accuracy for all groups for the first five transfer sessions is presented in Figure 2.

The major finding here is that there was no evidence of a drop in matching accuracy in the positive transfer groups, in spite of the fact that the differential outcome anticipations that presumably mediated high levels of transfer performance were disconfirmed by the actual nondifferential outcomes experienced in transfer.

\section{DISCUSSION}

The first goal of this experiment was to determine whether differential outcome expectancies alone (in the absence of potentially mediating differential-sampleresponding cues) could control comparison choice. Control by outcome anticipations was demonstrated by significant transfer effects in the three groups that could not use differential sample responding during transfer as the basis for comparison choice. Although in two of those groups differential sample responding could have contributed to acquisition of one or the other training tasks (i.e., the Diff-Resp/Nondiff-Resp group and the NondiffResp/Diff-Resp group), in neither case could that differential sample responding serve as an effective cue for comparison choice in transfer.

Thus, it appears that the natural peripheral consequences of differential outcome expectancies (i.e., differential sample responding) are not a necessary component of the DOE. Pigeons appear to use the expectancies themselves as cues for comparison choice, whether they have learned to make those choices in the context of available differential-responding cues (Diff-Resp/Nondiff-Resp group) or not (Nondiff-Resp/Diff-Resp and NondiffResp/Nondiff-Resp groups).

One might argue that requiring the pigeons to respond to both samples on an FR10 schedule may not have adequately eliminated differential responding. The fact that mean sample durations (time to complete the FR10) for the two sample types were virtually the same on the first transfer session for pigeons in the nondifferential responding groups suggests, at least, that the rate of sample responding was comparable for the two samples. Although it is possible that the response-rate measure is not sensitive enough to reveal a difference in the pattern or 


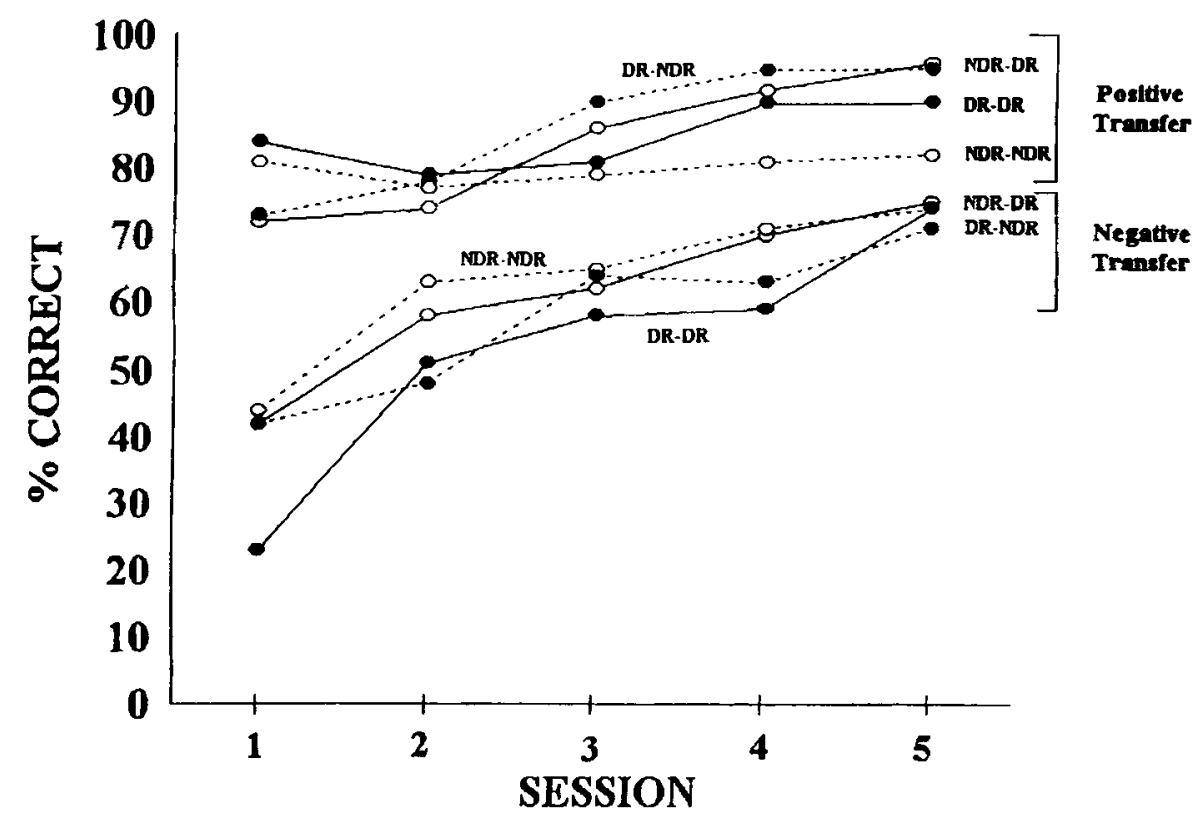

Figure 2. Matching accuracy for the first five transfer sessions. DR indicates that differential sample responding (10 responses to one sample, the absence of responding to the other) was required either in Phase 1 (left of dash) or in Phase 2 (right of dash). NDR indicates that nondifferential sample responding (10 responses to both samples) was required. In the positive transfer condition, the hypothesized outcome expectancies established during training were consistent with accurate responding. In the negative transfer condition, they were not (see Table 1).

topography of responding to the two samples, such undetected differential sample responding would have been capable of mediating transfer only in the Nondiff-Resp/ Nondiff-Resp group - the only group for which such differences in sample responding would have been common to both training phases. Differential sample responding could not have mediated transfer in either the Diff-Resp/ Nondiff-Resp group or Nondiff-Resp/Diff-Resp group, because, in these groups, the patterns of sample responding for the two phases were clearly dissimilar. The fact that the magnitude of the transfer effect in these groups was not significantly smaller than that in the NondiffResp/Nondiff-Resp group suggests that differential sample responding did not mediate transfer in the NondiffResp/Nondiff-Resp group, either. Thus the manipulation used to eliminate differential sample responding as a cue for comparison choice appears to have been effective.

A second goal of this experiment was to examine the contribution of differential sample responding to the DOE. A significantly larger transfer effect was shown in the group that could use differential sample responding to mediate transfer (the mean difference in performance between positive and negative transfer conditions for the Diff-Resp/Diff-Resp group was $60.5 \%$ correct) than in those that could not use such responding to mediate transfer (the mean difference in performance between the positive and negative transfer conditions for the other three groups combined was only $32.6 \%$ correct). This finding suggests that the spontaneously occurring, differential sample responding that has been observed in other differ- ential-outcomes experiments (see Brodigan \& Peterson, 1976; Urcuioli, 1990) may have contributed significantly to the effects found in those studies.

Examination of acquisition of the transfer task also merits comment. Consistent with the initial transfer results, pigeons transferred to the positive condition generally learned the task more quickly than those that were negatively transferred. For two of the groups that showed this effect, Diff-Resp/Nondiff-Resp and Nondiff-Resp/ Diff-Resp, this result is somewhat surprising, because the only basis for any initial transfer in these groups (differential outcome expectancies) was eliminated in the test phase. Apparently, sufficient learning of the new samplecomparison associations took place during early transfer trials for the level of accuracy to be maintained.

The positive transfer results may also provide a better understanding of why severe disruption of pigeons' conditional discrimination performance has been observed in several different experiments when differential outcomes for correct responding were (1) changed to mixed outcomes (Peterson, Wheeler, \& Armstrong, 1978), (2) reversed (Peterson et al., 1978), or (3) made the same for all correct responses (Peterson, Wheeler, \& Trapold, 1980). Typically, there is a substantial drop in matching accuracy for several sessions after the outcome change (see Peterson et al., 1978; Peterson et al., 1980). This decrement in matching accuracy has been interpreted as evidence for both the importance of expectancy cues and the overshadowing by outcome expectancies of other aspects of the sample stimuli in differential-outcomes tasks (see Urcuioli, 1990). 
As can be seen in the positive transfer acquisition functions shown in Figure 2, no comparable drop in matching accuracy was observed in the present experiment, in spite of the fact that not only were the differential outcomes eliminated, but also the comparison stimuli were replaced for these pigeons. In the Diff-Resp/Diff-Resp group, continued high levels of matching accuracy might have been expected because differential-sample-responding cues that had been available to mediate initial transfer performance continued to be available throughout testing. In the other three groups, however, the only apparent basis for abovechance transfer accuracy, differential outcome expectancies, had been eliminated. This absence of a drop in matching accuracy suggests that substantial new learning took place on early reinforced test trials. The learning must have been quite rapid, because it would have had to have taken place before differential outcome expectancies, the bases for those correct responses, were completely eliminated.

The absence of an expected drop in matching accuracy during the transfer phase in the present study may be due to the maintenance of the sample-response requirement present during acquisition. Although for three of the groups, the sample-response requirement could not be used as the basis for comparison choice, it could serve either to enhance sample discriminability (for the group for which it was differential) or at least to maintain consistent behavior in the presence of those samples (for the groups for which it was nondifferential). In the typical differential-outcomes experiment, it is unlikely that differential sample responding (which occurs spontaneously and appears to contribute to the DOE) is maintained when the outcomes are made nondifferential. Thus, it may be that the disruption in matching accuracy that is typically observed when differential outcomes are made nondifferential results, in part, from consequent spontaneous changes in sample responding.

In summary, the present results suggest that although differential sample responding contributes to the DOE, differential expectancies alone appear to be sufficient to produce the effect. The results reported here, together with those of Urcuioli and DeMarse (1994), indicate that pigeons can use either differential sample responding or differential outcome expectancies as the basis for comparison choice in a differential-outcomes conditional discrimination and that they are flexible enough to use the other when one set of cues is eliminated.

\section{REFERENCES}

Brodigan, D. L., \& Peterson, G. B. (1976). Two-choice conditional discrimination performance of pigeons as a function of reward ex- pectancy, prechoice delay, and domesticity. Animal Learning \& Behavior, 4, 121-124.

Cohen, L. R., Looney, T. A., Brady, J. H., \& Aucella, A. F. (1976). Differential sample response schedules in the acquisition of conditional discriminations by pigeons. Journal of the Experimental Analysis of Behavior, 26, 301-316.

Delong, R. E., \& WASSERMan, E. A. (1981). Effects of differential reinforcement expectancies on successive matching-to-sample performance in pigeons. Journal of Experimental Psychology: Animal Behavior Processes, 7, 394-412.

Edwards, C. A., JaGielo, J. A., Zentall, T. R., \& Hogan, D. E. (1982). Acquired equivalence and distinctiveness in delayed matching to sample by pigeons: Mediation by reinforcer-specific expectancies. Journal of Experimental Psychology: Animal Behavior Processes, 8, 244-259.

JENKINS, H. M., \& MOORE, B. R. (1973). The form of the auto-shaped response with food and water reinforcers. Journal of the Experimental Analysis of Behavior, 20, 163-181.

Peterson, G. B. (1984). How expectancies guide behavior. In H. L. Roitblat, T. G. Bever, \& H. S. Terrace (Eds.), Animal cognition (pp. 135-148). Hillsdale, NJ: Erlbaum.

Peterson, G. B., Wheeler, R. L., \& Armstrong, G. D. (1978). Expectancies as mediators in the differential-reward conditional discrimination performance of pigeons. Animal Learning \& Behavior, 6. 279-285.

Peterson, G. B., Wheeler, R. L., \& Trapold, M. A. (1980). Enhancement of pigeons' conditional discrimination performance by expectancies of reinforcement and nonreinforcement. Animal Learning \& Behavior, 8, 22-30.

Sherburne, L. M., \& Zentall, T. R. (1993). Asymmetrical coding of food and no-food events by pigeons: Sample pecking versus food as the basis of the sample code. Learning \& Motivation, 24, 141-155.

TRAPOLD, M. A. (1970). Are expectancies based on different reinforcing events discriminably different? Learning \& Motivation, 1, 129-140.

URCUIOLI, P. J. (1985). On the role of differential sample behaviors in matching-to-sample. Journal of Experimental Psychology: Animal Behavior Processes, 11, 502-519.

URCUIOLI, P. J. (1990). Some relationships between outcome expectancies and sample stimuli in pigeons' delayed matching. Animal Learning \& Behavior, 18, 302-314.

URCUIOLI, P. J., \& DEMARSE, T. (1994). On the relationship between differential outcomes and differential sample responding in matching-tosample. Journal of Experimental Psychology: Animal Behavior Processes, 20, 249-263.

URCUIOLI, P. J., \& HONIG, W. K. (1980). Control of choice in conditional discriminations by sample-specific behaviors. Journal of Experimental Psychology: Animal Behavior Processes, 6, 251-277.

Zentall, T. R., Hogan, D. E., Howard, M. M., \& Moore, B. S. (1978). Delayed matching in the pigeon: Effect on performance of sample-specific observing responses and differential delay behavior. Learning \& Motivation, 9, 202-218.

Zentall, T. R., \& Sherburne, L. M. (1994). The role of differential sample responding in the differential outcomes effect involving delayed matching by pigeons. Journal of Experimental Psychology: Animal Behavior Processes, 20, 390-401.
(Manuscript received March 31, 1994; revision accepted for publication August 16, 1994.) 